\title{
JUSTIFICACIÓN DE UNA PROPUESTA REGULATORIA PARA RADIODIFUSIÓN SONORA Y TRANSMÓVILES EN LAS FRONTERAS COLOMBIANAS
}

\author{
(Justification for a regulatory proposal for sound broadcasting and transmobile services in \\ Colombian borders)
}

Natalia Andrea Duarte Pinilla*, Hernán Paz Penagos **

*Departamento de Ingeniería Electrónica, Escuela Colombiana de Ingeniería Julio Garavito, na-duart@hotmail.com. ** Departamento de Ingeniería Electrónica, Escuela Colombiana de Ingeniería Julio Garavito, hpazster@gmail.com

(Recibido 26 de febrero 2015 y aceptado mayo 20 de 2015)

\begin{abstract}
Resumen:
Actualmente en Colombia no existe normatividad ni acuerdos que regulen los servicios de radiodifusión sonora y transmóviles en sus fronteras. Este artículo tiene como objetivo justificar la necesidad de reglamentar dichos servicios mediante un estudio de campo en tres zonas fronterizas: Ipiales-Tulcán (Ecuador); Puerto CarreñoPuerto Ayacucho (Venezuela) y Leticia-Tabatinga (Brasil)-Santa Rosa (Perú), para identificar posibles escenarios de interferencias procedentes de los países fronterizos hacia Colombia y viceversa. En este contexto, se realizaron simulaciones con el software Radio Mobile para evaluar la magnitud de las interferencias. Los resultados evidencian casos de interferencias entre servicios móviles y fijos, que demandan un control porque podrían llegar a afectar las comunicaciones destinadas a la seguridad de las personas y la atención de emergencias. Lo anterior también obstaculiza los procesos para la asignación de espectro y proyección de nuevos canales para los servicios de radiodifusión sonora y transmóviles.
\end{abstract}

Palabras clave: radiodifusión sonora, transmóviles, interferencia, espectro eléctrico, normatividad, zona de coordinación, organismos de regulación.

\begin{abstract}
:
Nowadays, in Colombia there are neither rules nor agreements that regulate transmobile and broadcasting sound services in its borders. This paper aims to justify the need to regulate these services through a study in three border areas: Ipiales-Tulcán (Ecuador); Puerto CarreñoPuerto Ayacucho (Venezuela) and Leticia-Tabatinga (Brazil)-Santa Rosa (Peru), to identify possible scenarios of interference from neighboring countries to Colombia.

In this context, we performed simulations with the software Radio Mobile to assess the magnitude of the interference. The results show cases of interference between mobile and fixed services, which require control because they could potentially affect communications to the safety and emergency care of people. This also hinders the process for allocating spectrum and screening of new channels for radio broadcasting services and transmobile.
\end{abstract}

Keywords: broadcasting services, transmobile, interference, radio spectrum, coordination area, regulation. 


\section{INTRODUCCIÓN}

La radiodifusión sonora es el servicio de voz y audio que satisface las necesidades de telecomunicaciones de los habitantes de una comunidad, región o país, cuyas emisiones se destinan al público en general (Mintic, 2008). En este escenario también se identifica el servicio de transmóviles, el cual consiste en una transmisión punto a punto o punto a zona desde una emisora hacia una unidad móvil o viceversa.

En Colombia los servicios de radiodifusión sonora y transmóviles para el interior del país están regulados por la Comisión de Regulación de Comunicaciones o CRC y por el Ministerio de Tecnologías de la Información y las Comunicaciones -Mintic- respectivamente. Estas entidades fundamentan su reglamentación en el cumplimiento de las normas internacionales, principalmente en las exigidas por la Unión Internacional de Telecomunicaciones o UIT.

En lo referente a normatividad para regular servicios de radiodifusión sonora y transmóviles para las fronteras en Colombia, no existen normas, solo hay un acuerdo multilateral regional para utilizar las bandas de frecuencia dispuestas para el servicio de radiodifusión sonora en el continente (UIT, 1981). De igual manera, esfuerzos aislados de los Gobiernos de turno, como un acuerdo bilateral entre Colombia y Ecuador que determina el uso compartido del espectro (Mintic, 2006).

En el contexto mundial, México, Estados Unidos y algunos países de la Unión Europea han adelantado acuerdos bilaterales relativos al servicio de radiodifusión sonora y transmóviles (IFT, 2015) con el ánimo de mejorar estos servicios en las zonas fronterizas. Lo anterior muestra que Colombia aún está atrasada en materia de regulación de los servicios mencionados arriba, ya sea por falta de información, voluntad o porque no ha visto la necesidad de ello.

En este artículo se recopilan los antecedentes del tema de estudio. Después se describen los servicios de radiodifusión sonora y transmóviles en Colombia; así mismo, se especifican las normas que regulan dichos servicios tanto a nivel nacional como internacional. Posteriormente se muestran los resultados obtenidos del trabajo de campo y simulación, así como su análisis e interpretación. Finalmente se justifica la necesidad de formular una propuesta de regulación para Colombia para los servicios de radiodifusión sonora y transmóviles para las fronteras.

\section{ANTECEDENTES Y MARCO TEÓRICO}

\subsection{Estudios realizados}

Dentro de los estudios que se han realizado en el tema de gestión del espectro radioeléctrico en radiodifusión sonora se encuentra el trabajo de Rico y Contreras (2012), que consiste en un estudio para determinar las características, las condiciones y las señales de interferencia a través de simulaciones, para los servicios de radiodifusión en el espectro radioeléctrico que puedan afectar su uso en los países vecinos. Los softwares utilizados para dicho proceso fueron GTOPO301 y SRTM. Las simulaciones se realizaron en las ciudades colombianas para AM y FM. No se hicieron simulaciones desde las ciudades de los países vecinos. Como resultado de este trabajo, las autoras destacan la necesidad de generar acuerdos entre los países fronterizos, con el ánimo de monitorear las emisiones del espectro radioeléctrico y calcular las pérdidas en la transmisión en las fronteras.

La Universidad Nacional de Colombia efectuó un estudio que permitió determinar las áreas de servicio y las características técnicas necesarias para la correcta operación de emisoras de radiodifusión sonora comunitaria en las ciudades capitales, según las bandas de frecuencia disponibles para la operación de estaciones de FM (Unal, 2007). Dentro de los resultados se encontró que la parametrización de una estación de FM típica debe tener potencias de máximo $100 \mathrm{~W}$ para servicios de radiodifusión comunitaria FM limitados a cobertura local, para el caso de las ciudades capitales con más de 1’000.000 de habitantes.

Otro estudio que se tuvo en cuenta fue el realizado por la Asociación para el Progreso de las Comunicaciones o APC, a cargo de Chamorro y Barbosa (2011). En este estudio se muestra la necesidad de proponer, discutir y participar en los temas de la gestión del espectro radioeléctrico en Colombia, por parte de la academia y otras instituciones, dado que las actuales estructuras que rigen estos procesos son muy nuevas y no conocen a fondo la problemática. Este trabajo justifica la necesidad de estudiar desde la academia los procesos actuales de gestión del espectro radioeléctrico. 
Un caso importante para destacar con respecto a los efectos que producen las interferencias de los servicios de radiodifusión en un país, es el de Haití y República Dominicana. Desde el 2010, el Gobierno de Republica Dominicana ha denunciado las interferencias que tiene en las zonas fronterizas con Haití. Hasta la fecha no se ha ejecutado ninguna acción que permita solucionar esta problemática. El estudio muestra que a la fecha, la interferencia cubre el $40 \%$ de la frontera, lo que eleva los perjuicios culturales, sociales, económicos y de seguridad del país (Impacto Digital, 2015). Esto justifica la necesidad de generar acuerdos bilaterales entre las diferentes administraciones que comparten zonas fronterizas para garantizar la buena gestión del ERE.

\subsection{Organismos y normatividad para radiodifusión so- nora}

La reglamentación que existente actualmente en Colombia para la gestión del espectro radioeléctrico en los servicios de radiodifusión sonora y transmóviles se fundamenta en la normatividad establecida por organismos internaciones tales como la UIT y la Comisión Interamericana de Telecomunicaciones o Citel (Citel, 2015).

La normativa de la UIT está contenida en un conjunto de documentos denominados recomendaciones, los cuales se agrupan como series. Las recomendaciones que están orientadas tanto al servicio de la radiodifusión sonora como a la gestión del espectro son las de la serie Broadcasting Service o BS y las Spectrum Management o SM. Se destacan las siguientes recomendaciones: UIT-R SM. 1049, UIT-R BS. 706-2, UIT-R BS. 703, UIT-R BS. 5592, UIT-R BS. 561-2, UIT-R BS. 598-1, UIT-R BS. 5604, UIT-R BS. 641, UIT-R BS. 467, UIT-R BS. 450-3, UIT-R BS. 599, UIT-R BS. 412-9.

Así mismo, la UIT ha creado un documento de carácter internacional conocido como el Reglamento de Radiocomunicaciones de la UIT. La última versión vigente corresponde al año 2012 (UIT, 2012). También se destacan documentos como el Acuerdo de Río o Acuerdo Regional sobre el Servicio de Radiodifusión Sonora por Ondas Hectométricas en la Región 2 (UIT, 1981) y el Manual Comprobación Técnica del Espectro (UIT, 2011).

\subsection{Convenios}

En materia de gestión del espectro radioeléctrico para los servicios de radiodifusión sonora y transmóviles en zonas fronterizas, el Gobierno colombiano ha promovido la generación de acuerdos con los vecinos países para el uso adecuado del espectro radioeléctrico. Hasta el momento solo se ha logrado un acuerdo con Ecuador. La Agencia Nacional del Espectro o ANE ha tenido acercamientos con las repúblicas de Venezuela y Brasil, pero no se evidencia aún ningún convenio o acuerdo en este aspecto.

Convenio con Ecuador en cuestiones de asignación y uso de frecuencias radioeléctricas para la operación de estaciones de radiodifusión sonora en la frontera:

El primer acercamiento con Ecuador fue en el año 2006 cuando se estableció el convenio en mención. Este fue instaurado por los ministerios TIC de cada país en diciembre de 2006, con el objetivo de definir el plan de distribución de frecuencias y los procedimientos de cooperación necesarios para la asignación y uso de canales de radiodifusión sonora y de televisión abierta VHF y UHF, a fin de lograr un servicio satisfactorio en sus respectivas zonas fronterizas (Mintic, 2006).

Bajo este convenio se determinó que para la radiodifusión sonora en AM, el rango de frecuencia es de 525 a $1.705 \mathrm{kHz}$ (ondas hectométricas). Para solucionar problemas de interferencia y de incompatibilidades en la asignación de frecuencias, se deben seguir los procedimientos definidos (UIT, 1981). Para el servicio en FM, el rango de frecuencia es de 88 a $108 \mathrm{MHz}$. Para la distribución de las frecuencias en la franja fronteriza se crearon seis grupos, tres para cada país.

\subsection{Acuerdos fronterizos para la asignación y uso de fre- cuencia radioeléctrica}

En febrero de 2015 se firmó un acuerdo modificatorio (Mintic, 2015) al convenio realizado en el 2006, con el fin de garantizar la operación libre de interferencias objetables de los sistemas de televisión. Para radiodifusión sonora en amplitud modulada o AM se estableció como rango de frecuencia de operación entre 525 a $1705 \mathrm{kHz}$ con una separación entre canales de $10 \mathrm{kHz}$. Para solucionar los problemas de incompatibilidad en la asignación de frecuencias y problemas de interferencia entre 
Ecuador y Colombia, se deben adoptar los procedimientos establecidos en UIT (1981). Para el caso de radiodifusión sonora en frecuencia modulada o FM, se definió el rango de frecuencia entre 88 a $108 \mathrm{MHz}$ con una canalización de 100 canales de $200 \mathrm{kHz}$ de ancho de banda cada uno. Para la distribución de las frecuencias entre los dos países se establecieron seis grupos, tres para las estaciones ubicadas en Ecuador y tres paras las estaciones ubicadas en Colombia.

Se acordó una zona de coordinación delimitada por una distancia de $15 \mathrm{~km}$ hacia cada uno de los respectivos países a partir de la línea de frontera. El valor de la intensidad de campo mínimo para proteger es de $54 \mathrm{~dB}(\mu \mathrm{V} / \mathrm{m})$. La potencia radiada y los transmisores de cada país deben ser tales que la intensidad de campo no sobrepase el valor mínimo.

En cuanto al manejo de interferencia se acordó que en el caso de producirse interferencias perjudiciales entre las estaciones de los dos países, la administración que se vea afectada debe solicitar a la otra identificar las causas y el origen técnico de estas, para así, poder aplicar los filtros correspondientes y realizar las modificaciones necesarias que eliminen la interferencia. El reporte debe hacerse según el apéndice 10 de la última versión vigente del Reglamento de Radiocomunicaciones de la UIT.

La señal de radiodifusión sonora cuya intensidad de campo sea superior a los establecidos por el acuerdo, se considerará como señal interferente y deberá ajustar sus características técnicas para que esto no siga sucediendo.

Para el servicio de transmóviles no se evidencian acuerdos.

\subsection{Servicios de radiodifusión sonora y transmóviles en Colombia}

A nivel nacional las principales entidades regulatorias que intervienen en los procesos de asignación y control del espectro electromagnético son el Ministerio de Tecnologías de la Información y las Comunicaciones, como ente supremo, y la Agencia Nacional del Espectro, como ente complementario.
2.6 Normatividad que regula los servicios de radiodifusión sonora y transmóviles

\subsubsection{En el interior}

La gestión eficaz del espectro a través de normas que permitan su regulación es un tema fundamental para garantizar el buen funcionamiento de los servicios de radiodifusión sonora o RDS y transmóviles en un país. Las normas concernientes están estipuladas en el Título VIII de la Ley 1341 de 2009.

a) $A M$ El Plan Nacional de Frecuencias para la Radiodifusión Sonora en Amplitud Modulada (Mintic, 2014) tiene como objetivo establecer el marco técnico que permita la adjudicación del mayor número de canales radioeléctricos posibles a los diferentes municipios y distritos del país, libres de interferencias objetables, de tal forma que se facilite la asignación de dichos canales y se racionalice el uso de este recurso, siguiendo los lineamientos del Reglamento de Radiocomunicaciones y las Recomendaciones de la UIT.

b) FM El Plan Nacional de Frecuencias para la Radiodifusión Sonora en Frecuencia Modulada (Mintic, 2014) comparte el mismo objetivo del Plan para la Radiodifusión Sonora en AM.

\subsubsection{Transmóviles}

La normativa del servicio de transmóviles se enuncia en el artículo 43 de la Resolución 415 de 2010 y en los artículos 6 y 7 de la Resolución 3120 de 2011 (Mintic, 2014).

\subsection{En las fronteras}

En cuanto a la regulación de este tipo de servicios en las fronteras, se evidencia una normativa incipiente en este aspecto, pues solo hay un convenio con Ecuador (Mintic, 2015).

Durante el 2013 se definió una propuesta técnica para el establecimiento de acuerdos bilaterales de frontera encaminados a facilitar el despliegue de servicios de radiocomunicaciones en zonas limítrofes (2013-2014). Así mismo, se formuló un proyecto para la gestión internacional del espectro radio eléctrico o ERE, con el objetivo de realizar actividades para armonizar el uso del espectro 
en Colombia tanto a nivel regional como internacional y de prevenir interferencias con otros países (ANE, 2013).

Para el 2014 se avanzó en el ámbito internacional en materia de gestión de espectro como banda ancha, espacios blancos, bandas de uso libre, esquemas de licenciamiento o autorización para el uso compartido de espectro en otros países y en organismos como la OECD, UIT, Citel $y$ entes reguladores (ANE, 2015).

Durante ese mismo año, en la XIII Cumbre del Comité Consultivo Permanente II (CCP.II) de la Comisión Interamericana de Telecomunicaciones, la ANE contribuyó con un documento de coordinación en zonas de frontera (Citel, 2015), en el que se recomienda considerar la adopción de medidas coordinadas oportunas, tendientes a viabilizar la utilización de la banda $698-806 \mathrm{MHz}$ por el servicio móvil terrenal, siempre en el contexto de no ocurrencia de interferencias perjudiciales en condiciones de frontera; así mismo se sugiere establecer acuerdos bilaterales o multilaterales sobre métodos unificados de comprobación técnica del espectro, como un medio para promover la utilización eficaz del espectro y luchar contra las interferencias en zonas fronterizas. También se recomienda que para los casos de interferencia perjudicial en zonas fronterizas, es conveniente contar con métodos de comprobación técnica del espectro estandarizados internacionalmente y homologados por los países involucrados, para encontrar rápidamente una solución (Ane, 2014).

\section{METODOLOGÍA}

Esta investigación reúne tres tipos de estudios: exploratorio, descriptivo y analítico. Estos estudios acuden a técnicas de recolección de información como la observación, las entrevistas, cuestionarios, informes de otros investigadores y el muestreo (Bernal, 2006; Méndez, 2001).

Los estudios de esta investigación se sustentan en leyes y decretos existentes, tanto a nivel nacional como internacional, sobre la gestión del espectro radioeléctrico para los servicios de radiodifusión sonora y transmóviles, así como en los acuerdos, recomendaciones y convenios que cobijan este tema en el ámbito fronterizo, para identificar, depurar y analizar las características que pueden generar interferencia perjudicial entre las estaciones prestadoras de estos servicios en las poblaciones limítrofes de Colombia con Ecuador, Venezuela, Perú y Brasil.
Para el desarrollo de este trabajo se hizo, en primer lugar, una revisión de la información actual sobre las normativas, convenios, tratados y recomendaciones que existen tanto a nivel nacional como internacional aplicados para las fronteras. Después se realizaron visitas a tres puntos fronterizos donde se incluyeron siete ciudades: tres colombianas, como Leticia, Puerto Carreño e Ipiales, y cuatro aledañas a estas, como Santa Rosa y Tabatinga en Perú y Brasil, Puerto Ayacucho en Venezuela y Tulcán en Ecuador; para evidenciar escenarios donde se puedan presentar posibles interferencias desde y hacia canales en la banda de servicios de radiodifusión sonora y servicios complementarios transmóviles. Para ello se aplicó un instrumento tipo encuesta a una parte de la población de las ciudades visitadas y a algunas emisoras de estas. Así mismo, se realizaron mediciones utilizando como instrumento de medida el analizador de espectros Tektronix MDO3000, en puntos dentro de cada una de las respectivas ciudades, así como en puntos sobre la línea fronteriza que divide los dos países; y se reconocieron los escenarios de interferencias. Esto se fundamentó con la simulación de la interferencia que se produce en estas zonas fronterizas por medio del software Radio Mobile (B.

Con la aplicación de los instrumentos descritos anteriormente se obtuvieron los hallazgos que se presentan a continuación.

\section{RESULTADOS}

\subsection{Frontera Colombia-Ecuador por el departamento de Nariño y la región de Carchi}

Las ciudades visitadas fueron Ipiales y Tulcán. De las encuestas aplicadas a la población colombiana se encontró que de 20 personas encuestadas, el $15 \%$ (tres personas) ha notado interferencia por lo menos alguna vez entre las emisoras de los dos países. En Ecuador, 12 de las 20 personas, es decir el $60 \%$ de la población, han notado interferencia especialmente en horas de la noche o cuando se va la luz. Con las encuestas aplicadas a las emisoras se encontró lo siguiente: la cobertura de las cuatro emisoras colombianas abarca poblaciones del vecino país. Para el servicio de transmóviles, la emisora Ondas del Sur 89.1 FM presenta interferencia con los radioteléfonos de los taxis. Así mismo, solo la emisora Rumba Estéreo manifiesta que sí ha tenido interferencia con la emisora Tropicana 103.7 FM de Ecuador y con otra emisora sintonizada en el dial 104.9; pero aclara

Revista Ingeniería, Investigación y Desarrollo Vol. 15 № 2, Diciembre, 2015 
que esto ocurre en los límites con la línea fronteriza, es decir, llegando al puente de Rumichaca. Para las emisoras ecuatorianas, se encontró que la cobertura de las seis emisoras encuestadas abarca poblaciones del vecino país. Para el servicio de transmóviles, ninguna emisora manifiesta presentar interferencias. De las seis emisoras, dos indican que han tenido interferencia con emisoras colombianas por lo menos una vez. Ondas Carchenses 1250 AM afirma que la interferencia se produce con la emisora Cultural Bolívar 1220 AM. Radio Tulcán 94.1 FM no recuerda con qué emisora fue, porque este sucedió hace mucho tiempo.

En las visitas de campo se recogieron datos que muestran que la emisora Cultural Bolívar de Ipiales transmite con una potencia mucho mayor que la de la emisora Ondas Carchenses. Esto significa que la cobertura es más grande. De esta forma, siguiendo los lineamientos del Plan Nacional de Radiofrecuencia AM, para una emisora que transmite a $10 \mathrm{~kW}$ con una intensidad de campo de 1250 $\mu \mathrm{V} / \mathrm{m}$, como lo es la Cultural Bolívar, la distancia del alcance del contorno de protección para evitar interferencias debe ser entre 44 y $55 \mathrm{~km}$, y el sitio donde se reportó la interferencia está dentro de estos límites. Esto permite entender el porqué la emisora Ondas Carchenses de Ecuador manifiesta presentar interferencias por parte de la emisora Cultural Bolívar de Colombia.

Con las simulaciones realizadas con el programa Radio Mobile ${ }^{\circledR}$, se obtuvo que la emisora Tropicana de Ecuador llega con mayor potencia a Colombia que a Ecuador, ya que la intensidad de campo hacia Ipiales es de 59.6 $\mathrm{dB} \mu \mathrm{V} / \mathrm{m}$, mientras que para Tulcán es de $53.9 \mathrm{~dB} \mu \mathrm{V} / \mathrm{m}$. Así mismo, en la Figura 1 se puede observar que el nivel aumenta (zona roja) en el límite fronterizo, lugar donde, según las encuestas, había una posible interferencia. Esto demuestra que la emisora Tropicana interfiere con la emisora Rumba Estéreo en el área de la línea fronteriza entre los dos países.

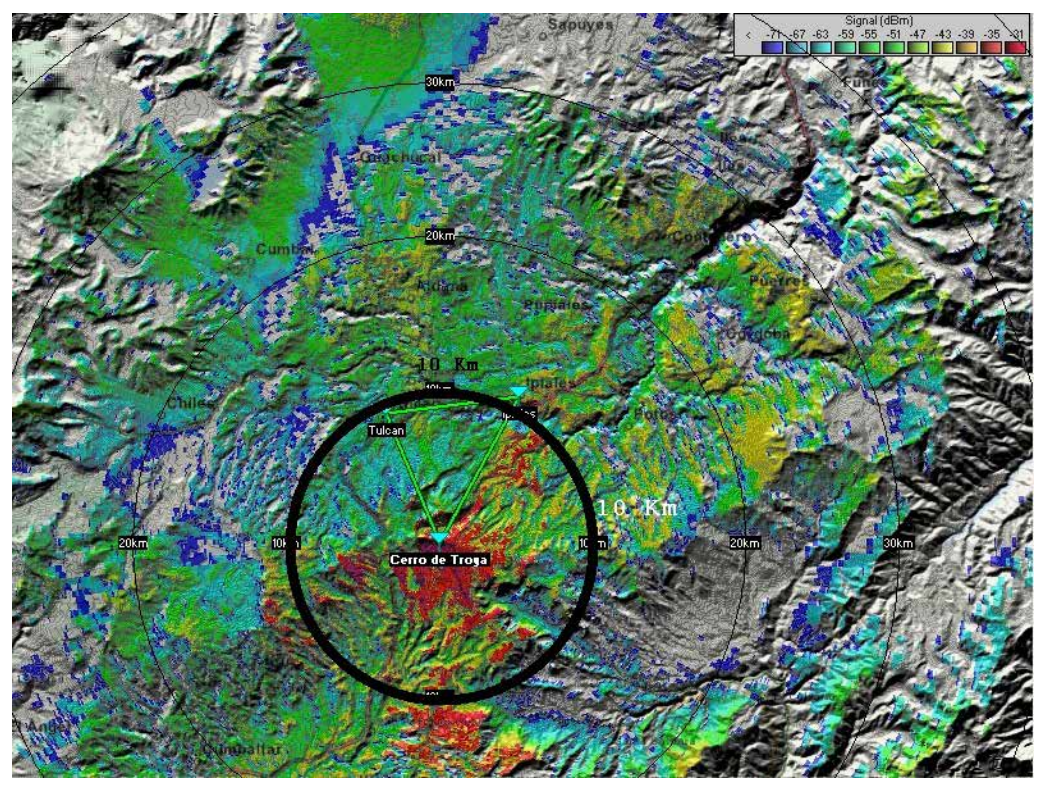

Figura 1. Cobertura Emisora Tropicana

\subsection{Frontera Colombia-Brasil-Perú por el departamen- to del Amazonas}

Las ciudades visitadas fueron Leticia, Tabatinga y Santa Rosa. De las encuestas aplicadas se encontró que en estas ciudades se sintonizan las tres emisoras respectivamente. Así mismo, las emisoras en Leticia afirman presentar interferencias entre las emisoras locales y la emisora brasilera para el servicio de transmóviles. En la emisora de Tabatinga se encontró que solo una vez ha tenido problemas de transmisión debido a interferencia con las emisoras colombianas. La emisora Activa Radio 91.1 FM manifiesta que en algunas ocasiones la emisora Tropicana 93.9 FM ha interferido con su señal, especialmente en horas de la noche, pero que esto no es constante. 
Para la construcción de este escenario de simulación se tuvieron en cuenta las coordenadas de cada una de las emisoras de las tres ciudades. Estos datos se tomaron desde la aplicación GoogleMaps, el cual utiliza el sistema de coordenadas geográficas mundiales World Geodetic System 84 o WGS84. Los datos técnicos de las emisoras colombianas se extrajeron de lo enunciado en el Plan Nacional de Radiodifusión FM. Los datos técnicos de las emisoras de Tabatinga y Santa Rosa se asumieron tomando como base los datos de las emisoras colombianas de los escenarios anteriores.

La Figura 2 muestra la cobertura de la emisora de Tabatinga. La Figura 3 indica la cobertura de la emisora de Leticia. Comparando las dos figuras, se puede observar que la emisora de Tabatinga tiene mayor cobertura que las otras, ya que su diámetro de cobertura sobrepasa los $10 \mathrm{~km}$, a diferencia de la emisora de Leticia que no pasa de este rango. Los valores de la intensidad de campo están dentro de los rangos permitidos: $77.3 \mathrm{~dB} \mu \mathrm{V} / \mathrm{m}$ para Tabatinga y $77.9 \mathrm{~dB} \mu \mathrm{V} / \mathrm{m}$ para Santa Rosa. Por lo tanto, según las simulaciones, no se evidencia interferencia por parte de ninguna de las emisoras.

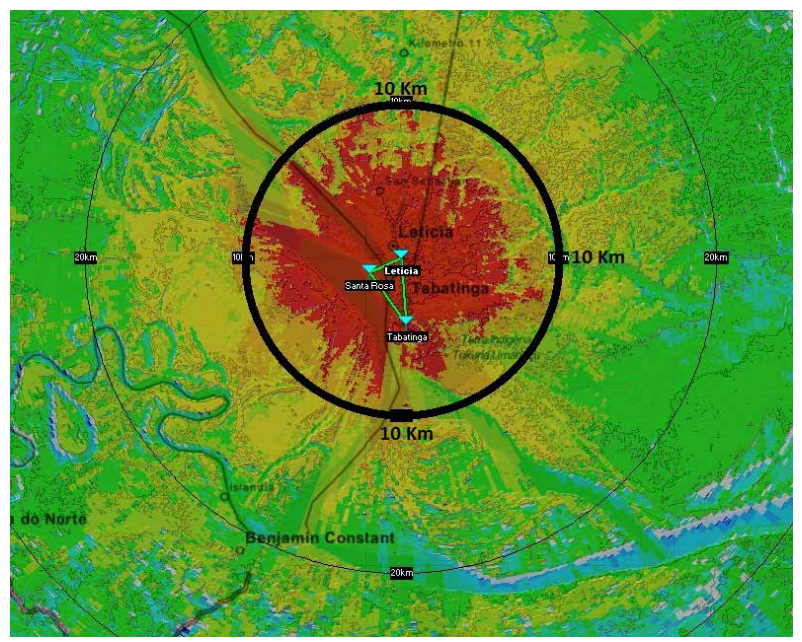

Figura 2. Cobertura Emisora Tropicana Estéreo de Leticia

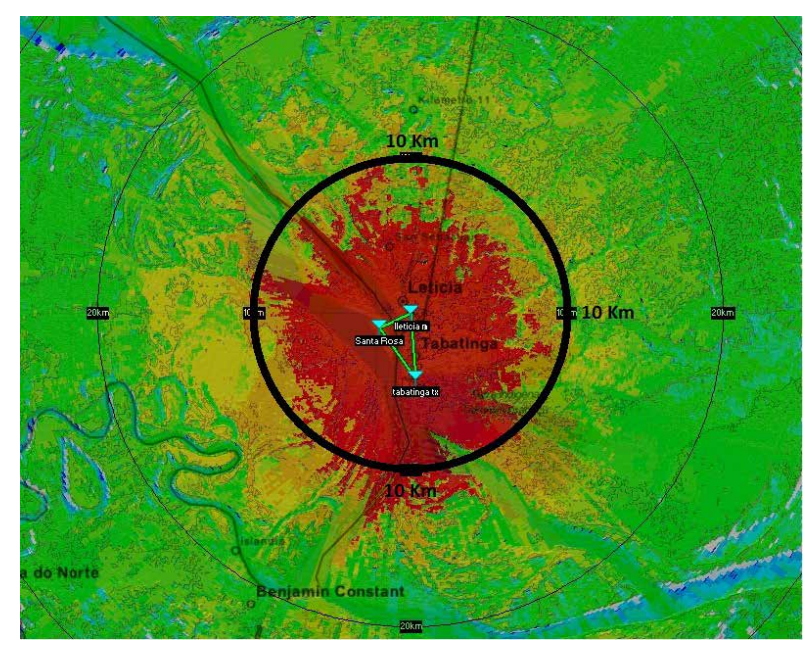

Figura 3. Cobertura Emisora Activa Radio de Santa Rosa, Perú, y Emisora Radio Nacional del Alto Solimoes de Tabatinga, Brasil

\subsection{Frontera Colombia-Venezuela por el departamento del Vichada}

Las ciudades visitadas fueron Puerto Carreño, Casuarito y Puerto Ayacucho. De las encuestas aplicadas a la población colombiana se encontró que en Puerto Carreño ninguna persona ha notado interferencia de las emisoras venezolanas. En Casuarito, dos personas que sintonizan la emisora colombiana manifiestan que a veces sí se presenta interferencia, pero no recuerdan con cuál emisora venezolana. Para las emisoras se encontró que la cobertura de las emisoras colombianas abarca poblaciones del vecino país. Para el servicio de transmóviles las emisoras manifiestan presentar interferencias entre ellas mismas. Así mismo, ninguna manifiesta presentar interferencias de las emisoras del vecino país. En Venezuela, las emisoras encuestadas manifestaron que tienen conocimiento de que sus señales llegan hasta las poblaciones de $\mathrm{Ca}$ suarito y Puerto Carreño en Colombia. La emisora Marawaka 103.1 FM mencionó que en algunas ocasiones la emisora colombiana Carreño Estéreo 103.7 FM ha interferido en su señal.

De los datos obtenidos con las mediciones de la emisora comunitaria Carreño Estéreo, esta está transmitiendo con una potencia mucho mayor que la permitida. Así mismo se identificó que esta emisora no se encuentra registrada dentro del Plan Nacional de Radiodifusión para FM. Lo cual puede llevar a pensar que esta emisora tiene un funcionamiento indebido que puede estar causando interferencia con alguna emisora del vecino país. 
En general, los resultados muestran que solo una de las emisoras de FM colombianas causa posiblemente interferencia a una emisora extranjera, porque está transmitiendo fuera de rango. Para los servicios de transmóviles y de radiodifusión sonora en AM no se evidencian interferencias. De los resultados hallados con el analizador de espectros, para el servicio de radiodifusión sonora en FM, las medidas muestran que en la mayoría de las emisoras no se presentan interferencias en ninguno de los países. Solo en dos emisoras se pudo observar presencia de interferencia, una colombiana y una extranjera. Esto se debe a las condiciones geográficas de la zona y al incumplimiento de los parámetros técnicos establecidos en el Plan Nacional de Radiodifusión en FM. De la misma manera, los resultados muestran que hay un posible funcionamiento de emisoras ilegales o clandestinas en Colombia. Para el servicio de transmóviles no se evidencia interferencia desde ni hacia Colombia.

Con relación a otros países, se encontró que países como México, Estado Unidos, Perú, Ecuador y la Unión Eu- ropea han avanzado en este tema considerablemente. Se evidencia que estas administraciones cuentan con leyes desde hace más de diez años, que regulan la buena gestión del espectro radioeléctrico para los servicios de RDS y transmóviles en las zonas fronterizas. Dentro de las recomendaciones está la necesidad de hacer un seguimiento continuo a las empresas o entidades prestadoras de los servicios de RDS y transmóviles en las fronteras, para reducir interferencias perjudiciales y evitar así problemas entre las administraciones. Así mismo, se recomienda que los Gobiernos tengan como prioridad mantener las buenas relaciones entre ellos a través de acuerdos y convenios que beneficien a ambas partes. De la misma manera, se sugiere crear entidades gubernamentales que se encarguen de realizar los estudios pertinentes o que a través de convenios con entidades académicas se gestionen nuevas investigaciones que contribuyan a la buena gestión del espectro radioeléctrico para los servicios en cuestión. En la Tabla 1 se muestra una correlación entre las normas internacionales y el caso colombiano para el servicio de radiodifusión sonora en FM:

Tabla 1. Correlación de recomendaciones de otros países aplicables a Colombia

\begin{tabular}{|c|c|c|}
\hline País & Acuerdo & Aplicación \\
\hline $\begin{array}{l}\text { México-Estados } \\
\text { Unidos }\end{array}$ & $\begin{array}{l}\left(\begin{array}{l}\text { S C T } \\
2015)\end{array}\right.\end{array}$ & $\begin{array}{l}\text { Existe una comunicación semestral entre las dos administraciones, en la cual se informa sobre } \\
\text { cualquier cambio, ajuste o nueva asignación que se haga. Así mismo, los dos países conocen entre } \\
\text { ellos los parámetros técnicos de cada una de sus estaciones. Colombia podía tomar esto como } \\
\text { ejemplo para hacer un mayor seguimiento en las estaciones fronterizas, así como, para mejorar las } \\
\text { relaciones con los países vecinos. } \\
\text { En el acuerdo en mención se define un procedimiento para determinar la Zona de Interferencia. Este } \\
\text { puede ser un buen aporte para nuevos tratados y acuerdos fronterizos. }\end{array}$ \\
\hline Perú-Ecuador & $\begin{array}{l}\text { ( M T C , } \\
\text { 2015) }\end{array}$ & $\begin{array}{l}\text { Este convenio es muy similar al firmado entre Colombia y Ecuador. De este convenio se puede } \\
\text { tomar como ejemplo la labor que ha realizado el Gobierno de Ecuador para generar acuerdo } \\
\text { binacionales con sus países vecinos. }\end{array}$ \\
\hline Unión Europea & $\begin{array}{l}\text { ( U I T , } \\
1995)\end{array}$ & $\begin{array}{l}\text { El Tratado de Viena es un modelo para la construcción de nuevos convenios. En este se recomienda } \\
\text { que las administraciones cuenten con una base de datos de las estaciones del vecino país. De la } \\
\text { misma manera, en este documento se especifican datos fundamentales que se pueden tener en } \\
\text { cuenta en el momento de realizar mediciones y que permiten detectar interferencias perjudiciales } \\
\text { entre dos o más canales de servicio de RDS y transmóviles. Así mismo, en el acuerdo se define un } \\
\text { procedimiento para realizar medidas para detectar interferencia, lo cual no se tiene establecido hasta } \\
\text { el momento en ningún documento nacional. }\end{array}$ \\
\hline
\end{tabular}

De los estudios realizados previamente, y a fin de continuar y complementar dichos trabajos, en esta investigación se incorporó el uso de una herramienta de simulación que permitiera comprobar los posibles escenarios de interferencia en las diferentes fronteras de Colombia. Así mismo, tomando como referencia los resultados obtenidos por Rico y Contreras (2012), se observa que para los servicios de radiodifusión sonora en AM en la frontera con Ecuador desde Ipiales y Tulcán hay una posible interferencia. Las autoras recomiendan hacer mediciones en terreno para verificar esto. Con los resultados obtenidos en esta investigación a través de los instrumentos aplicados en esta zona fronteriza se comprobó que existe una posible interferencia entre una emisora colombiana y una ecuatoriana. Para el departamento del Amazonas, las autoras no identifican interferencia, lo cual se comprobó en el presente estudio. Para el departamento del Vichada no se realizaron simulaciones ya que no hay 
emisoras AM en esta zona. En cuanto a los servicios de RDS en FM, las autoras encontraron que en el departamento del Amazonas no se evidencian interferencias, lo que también se demuestra en este estudio. Para el departamento de Nariño no se identificaron interferencias; sin embargo, los resultados del presente estudio evidencian interferencias tanto en las simulaciones como en las medidas. Para el departamento del Vichada no se realizaron simulaciones, lo cual sí se tuvo en cuenta en esta investigación.

Con respecto a las recomendaciones hechas por las autoras, ellas sugieren que Colombia debería pedir aspectos técnicos a los países de frontera con el fin de crear una base de datos que permita monitorear las emisiones del espectro radioeléctrico en zonas de frontera. En esta investigación se obtuvieron los parámetros técnicos de las emisoras de los vecinos países. Otra recomendación es revisar en un próximo estudio las emisiones generadas desde los países fronterizos hacia Colombia. Esto se hizo en el presente estudio. Finalmente, las autoras recomiendan que debe generarse una regulación para mitigar las interferencias radioeléctricas en zonas de fronteras, ya que a la fecha no se cuenta con dicha regulación.

La experiencia de otros países en este tema, evidencia que Colombia no cuenta con la normatividad suficiente para la gestión del espectro radioeléctrico para los servicios de radiodifusión sonora y transmóviles en las fronteras. Esto justifica la necesidad de crear una propuesta que fomente, impulse e inspire al Gobierno en la generación de nuevos acuerdos con los países vecinos.

\section{CONCLUSIONES}

La propagación de las ondas de radiocomunicaciones propias del espectro radioeléctrico no tiene límite ni fronteras, lo que hace necesario generar procesos de coordinación entre las administraciones para disminuir las interferencias perjudiciales en los sistemas de telecomunicaciones.

Los planes técnicos nacionales de radiodifusión sonora para AM y FM no incluyen especificaciones para los servicios de radiodifusión sonora y transmóviles en las zonas fronterizas.

El Gobierno de Colombia ha sumado esfuerzos para realizar acuerdos y convenios que permitan la buena ges- tión del espectro radioeléctrico para estos servicios en las fronteras.

Se evidencian casos de interferencia entre dos emisoras de las ciudades de Ipiales y Tulcán, así como un posible funcionamiento sin autorización de dos emisoras en Puerto Carreño, que además están generando interferencia en una emisora venezolana de la ciudad aledaña de Puerto Ayacucho.

En el campo internacional existen varios países que pueden servir de ejemplo para la generación de acuerdos y convenios en este tema.

Los siguientes documentos pueden servir de base para la generación de convenios y para la construcción de propuestas que permitan regular el uso del espectro radioeléctrico en los servicios de radiodifusión sonora y transmóviles en las fronteras: el acuerdo firmado por las administraciones de Colombia y Ecuador en febrero de 2015, el acuerdo regional firmado en Río en 1981 sobre el servicio de radiodifusión sonora por ondas hectométricas en la región 2, el acuerdo firmado en 1993 en Viena sobre la coordinación de frecuencias entre 29.7 y 960 $\mathrm{MHz}$ entre las autoridades de telecomunicaciones de 14 países europeos, la Recomendación UIT-R SM.1049-1 y la declaración de Citel en la XXV Reunión del Comité Consultivo Permanente II para Radiocomunicaciones en Medellín de 2015.

El acuerdo firmado por las administraciones de Colombia y Ecuador es un logro considerable para el buen uso del espectro radioeléctrico para los servicios de radiodifusión sonora y transmóviles en estas zonas de frontera; este puede servir de base para la construcción de nuevos acuerdos con el resto de países con los que Colombia comparte fronteras, tanto terrestres como marítimas.

El Acuerdo de Viena es un ejemplo aplicado por otros países que detalla la forma de impedir la interferencia perjudicial mutua y optimizar la utilización del espectro de frecuencias basándose en acuerdo mutuos.

Es necesario que el Gobierno de Colombia realice acuerdos con los países vecinos para la gestión eficiente del espectro radioeléctrico en los servicios de radiodifusión sonora y transmóviles en las zonas de frontera lo más pronto posible. 


\section{REFERENCIAS}

Ane. (2013). Plan estratégico 2013. Recuperado de http://www.ane.gov.co/index.php/component/ jdownloads/finish/3/505.html?Itemid $=0$

Ane. (2014). Gobierno de Costa Rica resalta gestión de la ANE. Recuperado de http://www.ane.gov.co/ index.php/costa-rica-resalta-la-labor-de-la-ane. html

Ane. (2015). Plan estratégico 2015. Recuperado de http://www.ane.gov.co/index.php/component/ jdownloads/finish/3/1044.html?Itemid $=0$

Bernal, C. (2006). Metodología de la investigación. México: Pearson.

Chamorro, L. \& Barbosa, A. (2011). Espectro abierto para el desarrollo: estudio caso Colombia. Bogotá: APC.

Citel. (2015). Comisión Interamericana de Telecomunicaciones. Recuperado de https://www.citel.oas. org/es/Paginas/default.aspx

Citel. (2015). XXI reunión del Comité Consultivo Permanente II: radiocomunicaciones incluyendo radiodifusión. Recuperado de https://www.citel.oas.org/en/SiteAssets/PCCII/Final-Reports/ P2!R-3323r1_e.pdf

Congreso de la República de Colombia. (2009). Ley 1341 de 2009. Recuperado de http://www.mintic.gov. co/portal/604/articles-3707_documento.pdf

IFT. (2015). Decreto de promulgación del Acuerdo entre el Gobierno de los Estados Unidos Mexicanos y el Gobierno de los Estados Unidos de América, relativo al Servicio de Radiodifusión en FM en la Banda de 88 a $108 \mathrm{MHz}$ y en AM en la Banda de 1605 a $1705 \mathrm{KHz}$. Recuperado de http://www. ift.org.mx/iftweb/industria-2/unidad-de-prospectiva-y-regulacion/internacional/acuerdos-bilaterales-con-estados-unidos-de-america/

Impacto Digital. (2015). Sánchez Roa afirma interferencia haitiana de radio y TV cubre el $40 \%$ de frontera. Recuperado de: http://impactodigital. com.do/?p=83215

Méndez, C. (2001). Metodología: diseño y desarrollo del proceso de investigación. Bogotá: McGraw-Hill.

Mintic. (2008). Decreto 2805 de 2008. Recuperado de http://www.mintic.gov.co/portal/604/articles-3530_documento.pdf

Mintic. (2006). Convenio entre Mintic y Conartel para la asignación y uso de frecuencia radioeléctri$c a$. Recuperado de http://archivo.mintic.gov.co/ mincom/documents/portal/documents/root/CovenioMinEcuador.pdf

Mintic. (2014). Plan técnico nacional de radiodifusión sonora en amplitud modulada (A.M.). Recuperado de http://www.mintic.gov.co/portal/604/ articles-4677_recurso_1.pdf

Mintic. (2014). Plan técnico nacional de radiodifusión sonora en frecuencia modulada. Recuperado de http://www.mintic.gov.co/portal/604/articles-4677_recurso_2.pdf

Mintic. (2014). Resolución 00415. Recuperado de http:// www.mintic.gov.co/portal/604/articles-3797_ documento.pdf

Mintic. (2015). Acuerdo modificatorio para la asignación y uso de frecuencias radioeléctricas para la operación e estaciones de radiodifusión sonora y de televisión en las fronteras. Medellín, Colombia: Mintic, Ministerios de Telecomunicaciones y de la sociedad de la información.

MTC. (2015). Convenio de asignación y uso de frecuencias radioeléctricas para la operación de estaciones de radiodifusión sonora y televisión VHF y UHF en el área de frontera. Recuperado de: http://www.peru.gob.pe/docs/PLANES/14018/ PLAN_14018_2014_parte2_opt.pdf

Rico, M. \& Contreras, D. (2012). Caracterización de las condiciones de interferencia en el espectro radioeléctrico para servicios de radiodifusión en 
zonas fronterizas de Colombia. Bogotá: Universidad de San Buenaventura.

SCT. (2015). Acuerdo entre el Gobierno de los Estados Unidos Mexicanos y los Estados Unidos de América, relativo al Servicio de Radiodifusión en F.M. en la banda de 88 a $108 \mathrm{MHz}$. Recuperado de http://www.sct.gob.mx/fileadmin/_migrated/ content_uploads/24_Acuerdo_entre_el_Gob de_los_EUM_y_los_EUA_relativo_al_ș

UIT. (1995). Recomendación UIT-R SM.1049-1. Recuperado de http://www.itu.int/dms_pubrec/itu-r/ rec/sm/R-REC-SM.1049-1-199510-I!!PDF-S. pdf

UIT. (1981). Actas finales de la Conferencia Administrativa Regional de Radiodifusión (Región 2).
Recuperado de http://www.itu.int/pub/R-ACTRRC.4-1981/es.

UIT. (2011). Manual de comprobación técnica del espectro. Recuperado de http://www.itu.int/dms pub/itu-r/opb/hdb/R-HDB-23-2011-PDF-S.pdf

UIT. (2012). Reglamento de radiocomunicaciones. Recuperado de: http://www.itu.int/net/about/legal-es.aspx

Unal. (2007). Estudio de propagación de FM para servicios de radiodifusión comunitaria en ciudades capitales de Colombia. Bogotá: Departamento de Ingeniería Eléctrica y Electrónica. Universidad Nacional de Colombia. 